\title{
Electrochemical Deposition of Nanowires in Porous Alumina
}

\author{
B. Kalska-Szostko ${ }^{a}$, E. Brancewicz ${ }^{a}$, P. Mazalski ${ }^{b}$, J. Sveklo $^{b}$, W. Olszewski ${ }^{b}$, \\ K. SZYMAŃSKI ${ }^{b}$ AND A. SIDOR ${ }^{a}$ \\ ${ }^{a}$ Institute of Chemistry, University of Białystok, Hurtowa 1, 15-399 Białystok, Poland \\ ${ }^{b}$ Institute of Experimental Physics, University of Białystok, Lipowa 41, 15-424 Białystok, Poland \\ Electrochemical deposition is a very efficient method for producing many types of modern materials. The \\ method is not expensive and does not have a limit for sample size. In our work the preparation of $\mathrm{Ni}$, Co and $\mathrm{Fe}$ \\ nanowires is presented. The obtained nanowires had different diameter and length which were tunable by template \\ porous material and time of deposition, respectively. The quality of the prepared wires was dependent also on \\ deposition mode. The smallest wires of the diameter around $40 \mathrm{~nm}$ were prepared in porous anodic alumina oxide \\ obtained from oxalic acid. The largest ones, around $120 \mathrm{~nm}$, were produced in phosphoric acid. The length could \\ be as large as the thickness of the oxide and reached up to about $1 \mu \mathrm{m}$. The morphology of wires was studied by \\ atomic force microscopy and scanning electron microscopy. The magnetic characterization was done with usage \\ of magnetic force microscopy and the Mössbauer spectroscopy. The wires show magnetization along their growth \\ direction.
}

PACS numbers: 82.45.-h, 62.23.Hj, 82.45.Qr, 81.07.-b, 75.75.+a, 81.07.-b

\section{Introduction}

Fast growth of the nanotechnology calls for low cost preparation of nanomaterials. Such a method is the electrochemistry which allows us to deposit materials in many different forms from the solutions. In this work the main attention will be paid to the structures obtained in porous anodic alumina templates. The morphology of the porous template strongly depends on the preparation conditions [1]. To study magnetic properties the Ni, Fe and Co wires were placed into pores of the different diameter. The magnetic properties of nanowires are strongly dependent of diameter and length of the structures as well as preparation conditions [2].

\section{Experimental and results}

The basic atomic force microscopy (AFM) images were taken with the use of NT-MDT Integra equipment. The magnetic force microscopy (MFM) studies were carried out after small changes of the AFM setup. Scanning electron microscopy (SEM) pictures have been obtained with the use of the Hitachi S-3000N microscope. The specially designed Mössbauer setup with the detection of the conversion electrons was involved in magnetic studies of the nanostructured films.

\subsection{Alumina matrix}

Templates of anodic alumina oxide (AAO) were prepared in solution of $0.2 \mathrm{M}(\mathrm{COOH})_{2}$ to obtain pores with size of about $40 \mathrm{~nm}$ (Fig. 1a,b). The larger holes in alumina were created with the use of $\mathrm{H}_{3} \mathrm{PO}_{4}$ and led to about $120 \mathrm{~nm}$ close packed less ordered structures which can be seen in Fig. 1c.
The particular anodization condition influences the shape of the holes, their density and packing. Current parameters and temperature were adjusted to obtain optimal in size and order of the pores templates, see Fig. 1. The pores seen by SEM and depicted in Fig. 1a are very small and due to this fact the AFM zoom is presented in Fig. 1b. (a)

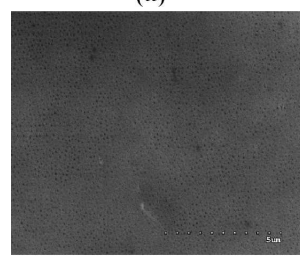

(b)

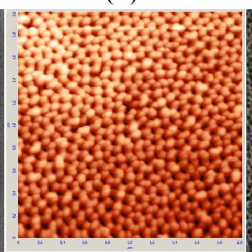

(c)

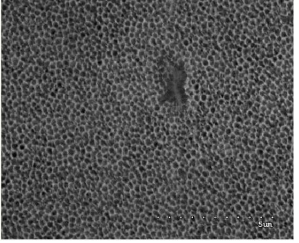

Fig. 1. SEM and AFM images of the porous alumina prepared in $(\mathrm{COOH})_{2}$ at $2^{\circ} \mathrm{C}, 40 \mathrm{~V}$ in a two-step process (a) and three-step process (b), respectively. SEM image of one step anodization in $\mathrm{H}_{3} \mathrm{PO}_{4}$ at $1^{\circ} \mathrm{C}, 40 \mathrm{~V}$ (c).

\section{2. $N i$}

The nanowires are prepared in the templates shown above. In case of the $\mathrm{Ni}$ deposition the constant current (DC) and alternating current (AC) deposition were done. The used electrolytes were composed from: aqueous solution of $120 \mathrm{~g} / 1 \mathrm{NiSO}_{4}$ and $40 \mathrm{~g} / \mathrm{l} \mathrm{H}_{3} \mathrm{BO}_{3}$ or acetate buffer $\mathrm{pH}=4,0.1 \mathrm{M} \mathrm{Ni}^{2+}$ [from $\left.\mathrm{Ni}\left(\mathrm{ClO}_{4}\right)_{2}\right]$, respectively for $\mathrm{AC}$ and DC deposition. The samples after deposition were bathed for $5 \mathrm{~min}$ in $5 \% \mathrm{H}_{3} \mathrm{PO}_{4}$. The final products seen by AFM and SEM are depicted in Fig. 2.

The SEM image shows very long wires (gray structures on the surface) which are sticking out of the matrix rather 
(a)

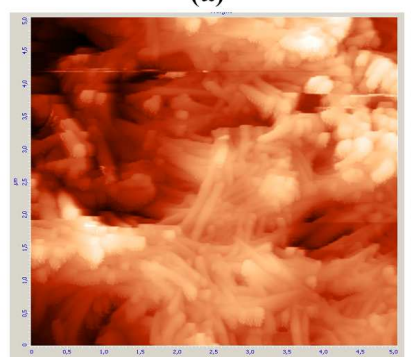

(c)

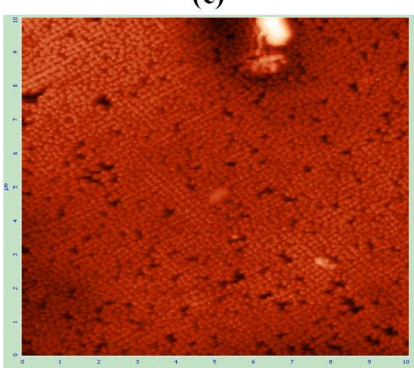

(b)

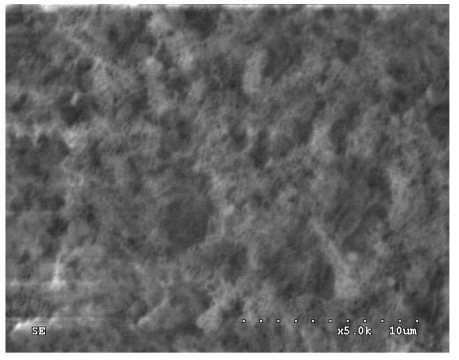

(d)

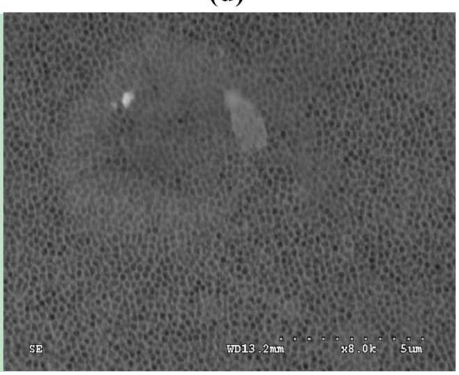

(a)

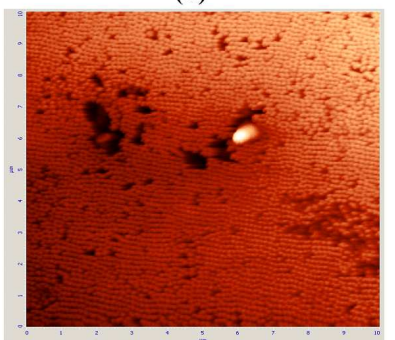

(c)

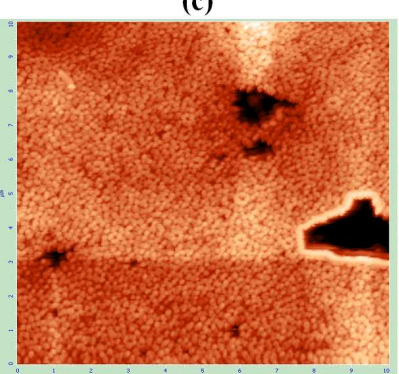

(b)

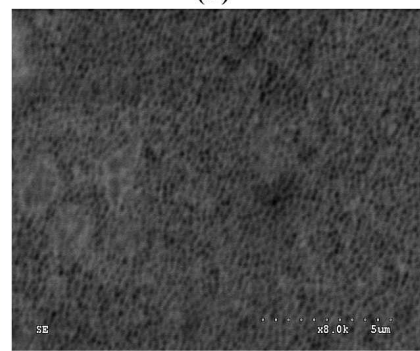

(d)

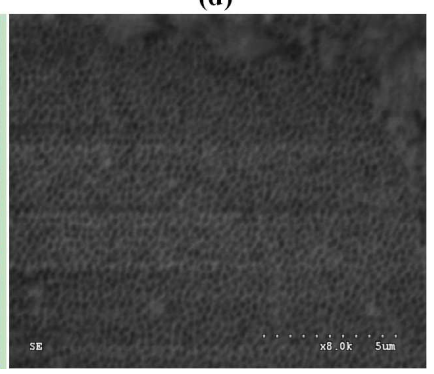

Fig. 3. As in Fig. 2, but for Co.

(a)

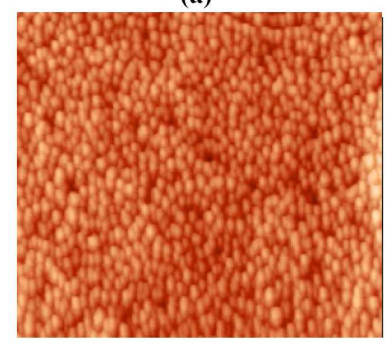

(c)

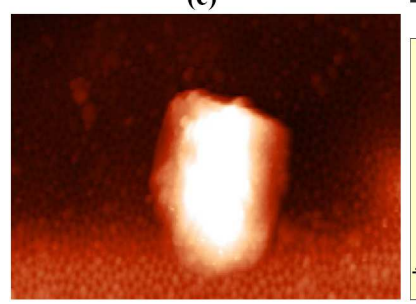

(b)

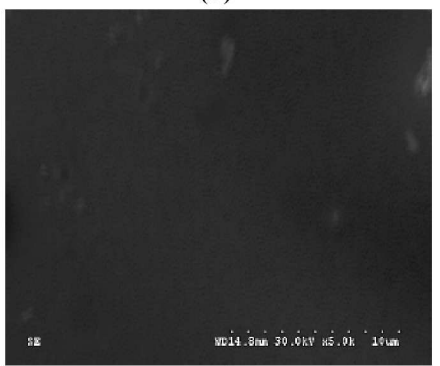

(d)

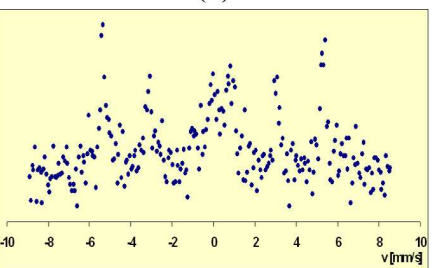

Fig. 4. The AFM (a) and SEM (b) images of Fe deposited in the AC mode. The AFM (c) image of the sample obtained in the DC mode; (d) Mössbauer spectrum for Fe nanowires. not merged together were obtained, see Fig. 3b. In case of $70 \mathrm{~min}$ DC deposition the island growth after filling up the pores can be seen (Fig. 3d, top-right corner). The deposition time in general in case of Co was longer than in Ni case to obtain the same length of the wires. The AFM images show that the wires are well organized on the surface and they are perpendicular to matrix plane.

\section{4. $\mathrm{Fe}$}

Fe deposition was done in mixture of: aqueous solution of $120 \mathrm{~g} / \mathrm{l} \mathrm{FeSO} \cdot 7 \mathrm{H}_{2} \mathrm{O}, 45 \mathrm{~g} / \mathrm{l} \mathrm{H}_{3} \mathrm{BO}_{3}, \mathrm{FeCl}_{3}, 1 \mathrm{~g} / \mathrm{l}$ ascorbic acid for both $\mathrm{AC}$ and $\mathrm{DC}$ processes. The samples after deposition were bathed for 5 min in $5 \% \mathrm{H}_{3} \mathrm{PO}_{4}$. The obtained wires are presented below in Fig. 4 .

The quality of the Fe nanowires is not so clearly seen on SEM picture (Fig. 4b) like in other cases because the template alumina had much smaller pores. Zoom in on the surface after AC deposition is presented in Fig. 4a. In case of Fe again is shown that the additional material in the form of round island on the surface can be seen only when DC mode is used, see Fig. 4a,c.

The results of deposition in form of nanowires shown in Figs. 2-4 brings us to general observation that the AC plating forms much more nanostructured material which is growing out of the pores in form of wires. The AC 
deposition is carried out in such way that after prior deposition part of the material is dissolved when the current direction is reversed.

In the DC deposition extra material tends to grow in islands rather than as continuous film of separated wires regardless the material.

\subsection{Magnetic measurements}

The magnetic properties of template with nanowires were studied by MFM and conversion electron Mössbauer spectroscopy in case of $\mathrm{Ni}, \mathrm{Co}$ and $\mathrm{Fe}$, respectively. By the comparison of the topographic images with the MFM maps (Fig. 5) we have obtained the confirmation of the deposition of magnetic material in the pores. The left part gives typical topographic images of studied samples. In the right one the magnetic force maps are presented. The changes of contrast in the MFM measurements are almost the same like in the topography photo. This means that the magnetic tip registry changes of the magnetic moments direction occurs exactly at the position of the nanowires.

(a)

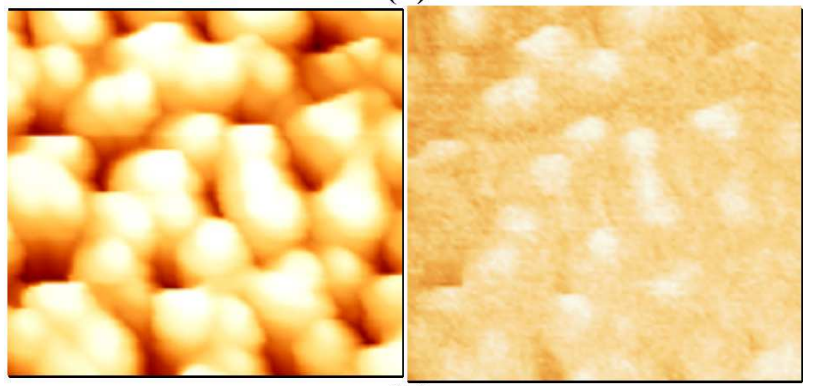

(b)

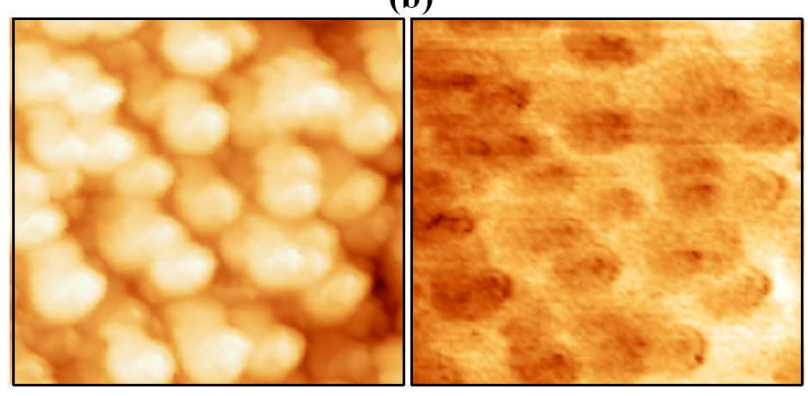

Fig. 5. MFM measurements of (a) Ni and (b) Co nanowires. Left part — topographic image, right phase contrast.

The line intensities in the Mössbauer spectrum are typical of preferred perpendicular orientation (Fig. 4d). In case of fully perpendicular orientation one expects 3:0:1:1:0:3 ratio, and nonvanishing line intensities (no. 2 and 5, Fig. 4d) show departure from perpendicular orientation. We interpret this result as indication that nanowires are magnetized along their axes, consistently with MFM results, and that part of nanowires lie on the surface consistently with result shown in Fig. 4c.
From the magnetic measurements we can conclude that the direction of easy axis in case of shown wires lies along the main axis of the nanowires which should be expected for configuration of the film. The detailed studies will be presented elsewhere [3].

\section{Conclusion}

The porous alumina templates were prepared in two kinds of solvents in oxalic and phosphoric acid. The anodization performed in oxalic and phosphoric acid leads to pores diameter 40 and 120, respectively. By electrodeposition $\mathrm{Ni}, \mathrm{Co}$ and $\mathrm{Fe}$ wires were obtained by $\mathrm{AC}$ plating as well as by $\mathrm{DC}$ deposition. In the $\mathrm{AC}$ plating the nanowires grow much longer than in DC deposition. From the magnetic investigations done by MFM can be concluded that magnetization direction lies along the main axis of nanowires.

\section{Acknowledgments}

The work was partially supported by UE MC ToK NANOMAG-LAB No. 2004-003177.

\section{References}

[1] H. Masuda, H. Tanaka, N. Baba, Chem. Lett. 19, 621 (1990); O. Jessensky, F. Muller, U. Gosele, J. Electrochem. Soc. 145, 3735 (1998); L. Ba, W.S. Li, J. Phys. D, Appl. Phys. 33, 2527 (2000); H. Maruda, F. Hesegwa, S. Ono, J. Electrochem. Soc. 144, L127 (1997); H. Maruda, K. Fukada, Science 268, 1466 (1995); H. Maruda, M. Satoh, Jpn. J. Appl. Phys. 35 , L126 (1996); S. Shingubara, O. Okino, Y. Sayama, H. Sakaue, T. Takahagi, Jpn. J. Appl. Phys. 36, 7791 (1997); A.-P. Li, F. Muller, A. Birner, K. Nielsch, U. Gosele, Adv. Mater. 11, 483 (1999); A.-P. Li, F. Muller, U. Gosele, Electrochem. Solid State Lett. 3, 131 (2000); A.-P. Li, F. Muller, A. Birner, K. Nielsch, U. Gosele, J. Vac. Sci. Technol. A 17, 1428 (1999); F. Li, L. Zhang, R.M. Metzger, Chem. Mater. 10, 2470 (1998).

[2] I.Z. Rahman, A. Boboc, K.M. Razeeb, M.A. Rahman, J. Magn. Magn. Mater. 290-291, 246 (2005); Cai-Ling Xu, Hua Li, Guang-Yu Zhao, Hu-Lin Li, Appl. Surf. Sci. 253, 1399 (2006); M. Zeng, L. Menon, H. Zeng, Phys. Rev. B 62, 12282 (2000); M. Vazquez, K. Pirota, J. Torrejon, D. Navas, M. HernandezVelez,J. Magn. Magn. Mater. 294, 174 (2005); H. Forster, T. Schrefl, W. Scholz, D. Suess, V. Tsiantos, J. Fidler J. Magn. Magn. Mater. 249, 181 (2002); R. Hertel, J. Kirschner, Physica B 343, 206 (2004); C.A. Ross, M. Hwang, M. Shima, H.I. Smith, J. Magn. Magn. Mater. 249, 200 (2002); H.Y. Zhang, X. Gu, X.H. Zhang, X. Ye, X.G. Gong, Phys. Lett. A 331, 332 (2004); P.M. Paulus, F. Luis, M. Kroll, G. Schmid, L.J. de Jongh, J. Magn. Magn. Mater. 224, 180 (2001); R. Hertel, J. Magn. Magn. Mater. 249, 251 (2002).

[3] B. Kalska, E. Brancewicz, W. Olszewski, K. Szymański, P. Mazalski, to be published. 